\title{
TOROIDAL MHD EQUILIBRIA AND CLASSICAL DIFFUSION IN COMPLEX MAGNETIC CONFIGURATIONS
}

\author{
H. M. RizK and T. KaMmash \\ Dept. of Nuclear Engineering, University of Michigan, Ann Arbor, Mich. 48105, U.S.A.
}

(Received 31 August 1974; and in final form 28 February 1975)

\begin{abstract}
Ideal MHD equilibrium for toroidal plasma situated in a magnetic field with single non-planar magnetic axis with variable torsion and curvature is investigated. The plasma is taken to have a non-circular cross section (elliptically or triangularly deformed) through which a longitudinal current with a flat profile flows. Equilibria of the Tokomak or French Harmonica types are examined as special cases of the general class and it is shown that for a given longitudinal current and torsion of the magnetic axis the effect of triangular deformation on the limiting value of $\beta$ (the ratio of kinetic to magnetic pressure) is weak and can be neglected. Vertically elongated elliptical deformation of the plasma cross section is shown to lead to an increase in the limiting value of $\beta$ relative to that of a circular cross section. Equilibria exist at a low value of $\beta$ in the absence of the longitudinal current. The particular type of stellarator with non-planar magnetic axis of constant torsion and with Ohmic heating is also considered. Moreover, classical diffusion of non-axisymmetric toroidal plasma is examined with the result that vertical elongation tends to decrease diffusion while triangular deformation appears to show an effect only at high $\beta$. In addition an expression for $V_{0}^{\prime \prime}$ is obtained and discussed for the existence of a magnetic well.
\end{abstract}

\section{INTRODUCTION}

DisTORTION of the plasma cross section as possible means of stabilizing plasmas in toroidal geometry and thus raising their betas has been intensively studied in recent years. Very recently (GLASSER et al., 1973) investigated the effect of such distortion on the collisionless trapped-particle instability in Tokamaks and have shown that elliptic deformations have a strong stabilizing influence while triangular distortions result in a much smaller effect. Moreover, several authors who have studied the equilibria and stability of Tokamaks (LAVAL et al., 1971) and the French Harmonica (ADAM and MERCIER, 1969) have shown that plasmas with non-circular cross section have deeper average magnetic wells than those with circular cross sections. In his study of plasma equilibrium and stability near the magnetic axis of complex toroidal configurations (MERCIER, 1964) has found that because of singularities no analytical solutions of the equilibrium equations exist. The position of this singularity depends on the ratio $j_{s 0} / B_{s 0}$ of the longitudinal components of the current density $j_{s 0}$ and the magnetic field $B_{s 0}$ near the magnetic axis, the rate $d^{\prime}(s) / 2$ at which the cross section turns around the magnetic axis and on the torsion $1 / T(s)$. The curvature $1 / R(s)$ of the magnetic axis has no influence on this position. For circular cross sections near the magnetic axis, Mercier has also shown that there is no domain of stability in the case of absence of longitudinal current (SHAFRANOv, 1968) and SOLEv'EV et al. (1969) have, however, shown that there indeed exists a domain of stability when the plasma cross section near the magnetic axis is taken as non-circular. Recently, Luc et al. (1974) have shown that by choosing some specific value of $\beta$ with elliptical cross section near the magnetic axis no domain of stability exists in the absence of longitudinal current and only the smallest stable value of longitudinal current is obtained with horizontal elliptical plasma cross section.

Moreover, an expression for the average velocity of diffusion across a magnetic surface of a stationary non-axisymmetric toroidal plasma near the magnetic axis has been obtained by MASCHKE (1971) using Mercier's coordinates and by NÜBERNBERG- 
(1972) using Hamada's coordinates but their results do not explain the resonance effects which appear near the magnetic axis.

In the first part of this work we examine the equilibrium of non-circular (elliptically or triangularly deformed) high beta toroidal plasma with a non-planar closed magnetic axis characterized by variable torsion and curvature. Of special interest is a toroidal configuration with or without a weak flat longitudinal current flowing in forward and backward directions in a plasma with non-circulator cross section. The resulting rotational transform is due to the current, the rotation of the noncircular cross section along the magnetic axis and the torsion in the magnetic axis. We find it especially useful to use the generalized Helicoidal image method (Luc et al., 1974) in order to avoid the difficulties associated with the singularities referred to earlier. We would like to point out that our equilibrium calculations here are for a thin cord plasma restricted near the magnetic axis using the so-called thin tube approximation and corresponding to the resonance case. Also, we have investigated the possibilities of existence of the magnetic well. The stability of this equilibria will be treated in a subsequent paper. A closely related investigation, namely that of the stability of a toroidal plasma with circular cross section but with different non-flat current profile will be treated in a later paper.

In the second part we derive a general expression for the average diffusion velocity across a magnetic surface near the magnetic axis and examine the resonance case. The results are then applied to various devices whose equilibria have been considered

\section{BASIC EQUATIONS AND ANALYSIS}

The magnetic ' $T$ ' is defined by its intrinsic coordinates $R(s)$ and $T(s)$ where $R(s)$ and $T(s)$ are respectively the radii of curvature and torsion expressed as functions of the curvilinear distance $s$. A point 'P' (See Fig. 1) is then defined by its polar coordinates $(\hat{\mu}, \theta)$ in a plaue normal to $\Gamma$ and the curvilinear coordinate ' $s$ '. Intruoducing $\theta_{0}=\theta+\alpha(s)$ where $\alpha(s)=\int_{0}^{s} \mathrm{~d} s / T(s)$ the square of the element of length is

$$
\mathrm{d} l^{2}=\mathrm{d} \rho^{2}+\rho^{2} \mathrm{~d} \theta_{0}^{2}+\left(1-\frac{\rho \cos \theta}{R(s)}\right)^{2} \mathrm{~d} s^{2} .
$$

The equations of interest are the ideal $\mathrm{MHD}$ equations: $\boldsymbol{\nabla} \cdot \mathbf{B}=0 ; \boldsymbol{\nabla} \times \mathbf{B}=\mathbf{j}$ and $\mathbf{j} \times \mathbf{B}=\nabla p$ where $\mathbf{B} ; \mathbf{j}$ and $\mathbf{P}$ are the magnetic field, current density and scalar pressure respectively. The solution of the first equation can be written with the aid of the helicoidal image method (Luc et al., 1974) as

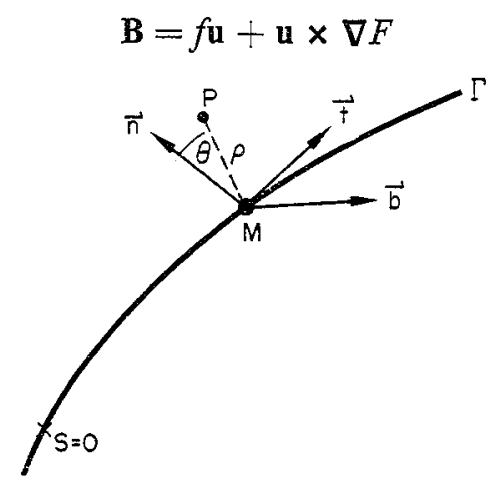

FIG. 1.-System of coordinates 
where $f$ and $F$ are function of $\rho$ and $t$, and the vector $\mathbf{u}$ is given by

$$
\mathbf{u}=\frac{1}{g}\left(h_{k} \mathbf{e}_{\mathbf{s}}+\frac{\hat{\varepsilon}_{t}}{\alpha} \rho \mathbf{e}_{\theta_{0}}\right) .
$$

In the above equations the following definitions have been employed;

$$
\begin{gathered}
t=\theta+\frac{\delta(s)}{2} ; \quad \frac{\delta(s)}{2}=\varphi_{k_{0}}+\alpha(s)-\frac{\varepsilon_{t} s}{\alpha} ; \\
g=h_{k}^{2}+\left(\frac{\varepsilon_{t} \rho}{\alpha}\right)^{2} ; \quad h_{k}=\left(1-\frac{\varepsilon_{k}}{\alpha} \rho \cos t\right) ; \quad \varepsilon_{t}=-\varepsilon_{0} k_{t} ; \\
\varepsilon_{k}=\alpha A_{k_{0}} ; \quad k_{t}=k_{0}-\frac{\alpha(L)}{2 \pi} ; \quad \varepsilon_{0}=\frac{2 \pi \alpha}{L}
\end{gathered}
$$

is the inverse of the aspect ratio, $a$ is the characteristic dimension of the plasma cross section. $L=\oint \mathrm{d} s$ is the total length of the magnetic axis, and $A_{k_{0}}$ is the resonant coefficient corresponding to the index $k_{0}$ related to the Fourier expansion of the relative curvature of the non-planar magnetic axis as given by (SHAFRANOv, 1964)

$$
\frac{\exp \left[i\left(\frac{\alpha(L)}{L} s-\alpha(s)\right)\right]}{R(s)}=\sum_{k=-\infty}^{+\infty} a_{k} \exp \left(\frac{i 2 \pi k_{s}}{L}\right)
$$

with the coefficient $a_{k}$ being given by

$$
a_{k}=\frac{1}{L} \int_{0}^{L} \frac{\mathrm{d} s}{R(s)} \exp \left[i\left(\frac{\alpha(L)}{L} s-\alpha(s)-\frac{2 \pi k_{s}}{L}\right)\right]
$$

Here $a_{k} \neq a_{-k}$ is a complex number that can be expressed as $a_{k}=A_{k} \exp \left(i \varphi_{k}\right)$ ( $a_{k}=a_{-k}$ for planar magnetic axis). It is important to note (LuC et al., 1974) at this point that there is one and only one $A_{k_{0}}$ coefficient that can be dominant in the equilibrium, stability and classical diffusion problems of this type of complex magnetic toroidal configuration. The expression for the current density can also be given in terms of the above functions as

$$
\mathbf{J}=\left(\mathscr{L} F+\frac{2 \varepsilon_{t} f}{a g}\right) \mathbf{u}+\mathbf{u} \wedge \nabla(-f)
$$

where the operator $\mathscr{L}$ is given by

$$
\mathscr{L}=\frac{g}{\rho h_{k}}\left[\frac{\partial}{\partial \rho}\left(\frac{\rho h_{k}}{g} \frac{\partial}{\partial \rho}\right)+\frac{\partial}{\partial t}\left(\frac{1}{\rho h_{k}} \frac{\partial}{\partial t}\right)\right] .
$$

In view of this equation for MHD equilibrium then assumes the form (LUC et al., 1974)

$$
\mathscr{L} F+\frac{2 \varepsilon_{t} f}{a g}+\frac{1}{2} \frac{\mathrm{d} f^{2}}{\mathrm{~d} F}+g \frac{\mathrm{d} P}{\mathrm{~d} F}=0
$$

and one can readily show that the function $f$ as well as the pressure $P$ are arbitrary functions of $F$. We shall assume in the present calculation that the plasma is a thin 
chord with $\varepsilon_{k} \sim \varepsilon_{t} \sim \varepsilon_{0} \sim \varepsilon \ll 1$ (the so-called 'thin tube' approximation) so that all terms containing $\varepsilon$ can be neglected relative to unity in the zero order terms. We will assume that $\left|\rho_{m}\right| / a<1$ to keep the magnetic axis always inside the plasma; here $\rho=\rho_{m}$ and $t=\pi$ are the coordinates of the position of the magnetic axis relative to the central geometrical axis.

We turn now to the calculation of the magnetic surface functions for configurations with closed, non-planar magnetic axis with variable torsion and curvature. The volume of a bounded magnetic surface, $F=$ constant, can be written as

$$
V=\int_{0}^{L} \mathrm{~d} s \int_{0}^{2 \pi} \mathrm{d} \theta \int_{0}^{\rho(s)} \mathrm{d} \rho \rho\left(1-\frac{\rho \cos \theta}{R(s)}\right) .
$$

Moreover, the short way (transverse) fluxes $\Psi$ and $\mathrm{X}$ of the magnetic field and the current density are given by

$$
\begin{gathered}
\Psi=\int_{0}^{L} \mathrm{~d} s \int_{0}^{\rho(s)} \mathrm{d} \rho\left[B_{\theta_{0}}\left(1-\frac{\rho \cos \theta}{R(s)}\right)-B_{s} \frac{\rho}{T}\right] \\
\chi=\int_{0}^{L} \mathrm{~d} s \int_{0}^{\rho(s)} \mathrm{d} \rho\left[j_{\theta_{0}}\left(1-\frac{\rho \cos \theta}{R(s)}\right)-j_{s} \frac{\rho}{T(s)}\right]
\end{gathered}
$$

where $B_{s}, B_{\theta_{0}}$ and $j_{s}, j_{\theta_{0}}$ are respectively the longitudinal and azimuthal components of both the magnetic field and current density. By the same token the long way (longitudinal) fluxes $\bar{\Psi}$ and $\bar{\chi}$ of the magnetic field and current density are represented by

$$
\begin{gathered}
\bar{\Psi}=\int_{0}^{2 \pi} \mathrm{d} \theta \int_{0}^{\rho(\theta)} \mathrm{d} \rho\left(B_{s} \rho\right) \\
\bar{\chi}=\int_{0}^{2 \pi} \mathrm{d} \theta \int_{0}^{\rho(\theta)} \mathrm{d} \rho\left(j_{s} \rho\right) .
\end{gathered}
$$

For planar magnetic axis i.e. $1 / T(s)=0$, equations (5b) and (5d) reduce to those obtained by MERCIER (1966). It might be noted that the two flux functions $\Psi$ and $\bar{\Psi}$ and the rotational transform $i_{c} / 2 \pi=\mathrm{d} \Psi / \mathrm{d} \bar{\Psi}$ are related to lowest order by

or

$$
\Psi=-k_{0} \bar{\Psi}+L\left(F-F_{m}\right)
$$

$$
\frac{i_{\llcorner 0}}{2 \pi}=-k_{0}+L \frac{\mathrm{d} F}{\mathrm{~d} \bar{\Psi}}
$$

where $F_{m}$ is the value of the function $F$ on the magnetic axis. The expression for the rotational transform near the magnetic axis (Luc et al., 1974) is given by

$$
\frac{i_{c 0}}{2 \pi}=-k_{0}+\frac{L K(s)}{2 \pi}
$$

where the function $K(s)$ has the form

$$
\begin{aligned}
K(s) & =\frac{1}{\operatorname{ch} \eta(s)}\left[\frac{j_{s 0}}{2 B_{s 0}}+\frac{\mathrm{d}^{\prime}(s)}{2}-\frac{1}{T}\right] \\
& =\sqrt{1-E_{m}^{2}}\left(\frac{j_{s 0}}{2 B_{s 0}}-\frac{\varepsilon_{t}}{a}\right)
\end{aligned}
$$


and $\eta(s)$ is related to the ellipticity parameter $E_{m}=\left(b_{m}{ }^{2}-a_{m}{ }^{2}\right) /\left(b_{m}{ }^{2}+a_{m}{ }^{2}\right)$ of the non-circular plasma cross section near the magnetic axis through the relation $E_{m}=$ $t h \eta(s)$. The terms $2 b_{m}$ and $2 a_{m}$ are respectively the major and minor axis of the small ellipse near the magnetic axis, $\mathrm{d}^{\prime}(s) / 2=\mathrm{d} / \mathrm{d} s(\mathrm{~d}(s) / 2)$, and $\mathrm{d}(s) / 2=\varphi_{K_{0}}+\alpha(s)-$ $\varepsilon_{t} S / a$ is the turning angle of the minor axis of the ellipse relative to the principal normal to the magnetic axis. From $(6 a),(7 a$ and $7 b)$ one can obtain the value of the function $\mathrm{d} F / \mathrm{d} \bar{\Psi}$. Accordingly, the function $P_{0}{ }^{\prime}=\left(\mathrm{d} P / \mathrm{d} \Psi^{\prime}\right)_{0}$ is given directly by

$$
P_{0}^{\prime}=\frac{\sqrt{1-E_{m}^{2}}}{2 \pi}\left(\frac{j_{s 0}}{2 B_{s 0}}-\frac{\varepsilon_{t}}{a}\right)\left(\frac{2 \pi}{i_{c 0}}\right) \frac{\mathrm{d} P_{(F)}}{\mathrm{d} F} .
$$

Similarly the relation between $x$ and $\bar{x}$ and the rational transform $\sigma_{c} / 2 \pi=\mathrm{d} x / \mathrm{d} \tilde{x}$ is given by

or

$$
x=-k_{0} \bar{x}+L\left(f-f_{m}\right)
$$

$$
\frac{\sigma_{c}}{2 \pi}=-k_{0}+L \frac{\mathrm{d} f}{\mathrm{~d} \bar{x}}
$$

where $f_{m}$ is the value of $f$ on the magnetic axis.

Through an expansion to third order in $\bar{\rho}$, the distance from the magnetic axis, the function $\Psi$ can be expressed in two well known forms, the so-called standard form (MERCIER, 1963) given by

$$
\frac{2 \Psi}{\left|i_{c_{0}}\right| B_{s_{0}}}=\tilde{\rho}^{2}(\operatorname{ch} \eta+\operatorname{sh} \eta \cos 2 u)+\tilde{\rho}^{3}\left(\sqrt{B_{s_{0}}} \tilde{r} \cos 3 u+\sqrt{B_{s_{0}}} \tilde{s} \sin 3 u\right)
$$

and the so called normal form (SOLEV'Ev and SHAFRANOv, 1970) denoted by

$$
\begin{aligned}
\Psi & =\frac{\pi B_{s_{0}}\left|\frac{i_{c 0}}{2 \pi}\right|}{\sqrt{1-E_{m}^{2}}} \\
& \times\left[\tilde{\rho}^{-2}\left(1+E_{m} \cos 2 u\right)+\tilde{\rho}^{3}\left(\alpha_{1} \cos u+\alpha_{2} \sin u+\alpha_{3} \cos 3 u+\alpha_{4} \sin 3 u\right)\right]
\end{aligned}
$$

where $u=\theta+\mathrm{d}(s) / 2$ is the phase angle of the magnetic surface near the magnetic axis which has a period of $2 \pi$. The function $\tilde{r}$ and $\tilde{s}$ which appear in (12) give the corrections to the elliptic form of the magnetic surfaces near the magnetic axis. These two functions are important because they appear in the necessary stability condition (MERCIER, 1964) near the magnetic axis. The functions $\alpha_{1}, \alpha_{2}, \alpha_{3}$ and $\alpha_{4}$ are related to one another (SOLVEV'EV and SHAFRANOV, 1970) and also enter into the necessary and sufficient stability condition (SOLVEV'EV, 1968, 1969) near the magnetic axis as well as in the definition $V_{0}^{\prime \prime}=\mathrm{d}^{2} V / \mathrm{d} \bar{\Psi}^{2}$ which characterizes the curvature of the mean magnetic well. The relations connecting the parameters $\tilde{r}$ and $\tilde{s}$, and $\alpha_{1}, \alpha_{2}, \alpha_{3}$ and $\alpha_{4}$ are given in the third approximation by

and

$$
\sqrt{B_{s 0}} \tilde{r}=\frac{\alpha_{3}\left(2+E_{m}\right)-\alpha_{1} E_{m}}{\sqrt{1-E_{m}^{2}}\left(2+E_{m}\right)}
$$

$$
\sqrt{B_{s 0}} \tilde{s}=\frac{\alpha_{4}\left(2-E_{m}\right)-\alpha_{2} E_{m}}{\sqrt{1-E_{m}^{2}}\left(2-E_{m}\right)}
$$


The expression for the function $V_{0}^{\prime \prime}$ near the magnetic axis has the form (SOLVEV'EV and SHAFRANOV, 1970);

$$
\begin{aligned}
V_{0}^{\prime \prime}= & -\frac{1}{\pi} \int_{0}^{L} \frac{\mathrm{d} s}{B_{s 0}^{2} \sqrt{1-E_{m}^{2}}}\left(\frac{1}{2 R^{2}(s)}\left[1-E_{m} \cos \mathrm{d}(s)\right]-\frac{3}{4}\left(\frac{B_{s 0} 0^{2}}{B_{s 0}}\right)^{2}-\frac{\eta^{\prime 2}(s)}{4}\right. \\
& -E_{m}^{2}\left(\frac{\mathrm{d}^{\prime}(s)}{2}-\frac{1}{T(s)}\right)^{2}+\frac{E_{m} \eta^{\prime}(s) B_{s 0}^{\prime}}{B_{s 0}} \\
- & {\left[\frac{\left(2-E_{m}\right) \alpha_{1}-3 E_{m} \alpha_{3}}{1+E_{m}}\left(\frac{\cos \frac{\mathrm{d}(s)}{2}}{R(s)}\right)+\frac{\left(2+E_{m}\right) \alpha_{2}-3 E_{m} \alpha_{4}}{1-E_{m}}\left(\frac{\sin \frac{\mathrm{d}(s)}{2}}{R(s)}\right)\right] . }
\end{aligned}
$$

It might be noted that this expression for $V_{0}^{\prime \prime}$ does not explain the resonance case, and in order to calculate $V_{0}^{\prime \prime}$ for the resonance case one needs to use the integrals (Luc et al., 1974) given by

$$
\begin{gathered}
\int_{0}^{L} \frac{\mathrm{d} s}{R^{2}(s)}=L \sum_{k=-\infty}^{+\infty}\left|A_{k}\right|^{2} ; \quad \int_{0}^{L} \mathrm{~d} s \frac{\cos \frac{\mathrm{d}(s)}{2}}{R(s)}=L A_{k_{0}} \\
\int_{0}^{L} \mathrm{~d} s \frac{\cos \mathrm{d}(s)}{R^{2}(s)}=L \sum_{k=-\infty}^{+\infty} A_{k} A_{2 k_{0}-k} \cos \left(\phi_{k}-\phi_{2 k_{0}-k}-2 \phi_{k_{0}}\right) ; \quad \int_{0}^{L} \mathrm{~d} s \frac{\sin \frac{\mathrm{d}(s)}{2}}{R(s)}=0 .
\end{gathered}
$$

The expression for $V_{0}^{\prime \prime}$ which yields the resonance case for the configuration with non-planar magnetic axis of variable curvature and torsion, in which the functions $\eta(s), j_{s 0}$ and $B_{s 0}$ are constant, is given by

$$
\begin{array}{r}
V_{0}^{\prime \prime}=-\frac{L}{\pi B_{s 0}{ }^{2} \sqrt{1-E_{m}^{2}}}\left\{\frac{1}{2}\left(\sum_{k}\left|A_{k_{0}}\right|^{2}-E_{m} \sum_{k} A_{k} A_{2 k_{0}-k} \cos \left(\phi_{k}-\phi_{2 k_{0}-k}-2 \phi_{k_{0}}\right)\right)\right. \\
\left.-\frac{E_{m}{ }^{2} \varepsilon_{t}^{2}}{\alpha^{2}}-\left(\frac{\left(2-E_{m}\right) \alpha_{1}-3 E_{m} \alpha_{3}}{1+E_{m}}\right) A_{k_{0}}\right)
\end{array}
$$

\section{EQUILIBRIUM}

\section{i. Equilibrium solutions}

To obtain a solution of the MHD equilibrium equation for non-circular (elliptically or triangularly deformed) cross section in the complex field geometry referred to earlier it is important to first note that to lowest order the central and magnetic axes are so close to each other that they can be assumed to possess the same length, torsion and curvature. We also note that a longitudinal current density with a flat distribution can be represented by $f^{2}=f_{0}{ }^{2}+\lambda F$ where $\lambda$ and $f_{0}$ are constant parameters. The function $f$ has been expanded to yield $f=f_{0}+0\left(\varepsilon^{2}\right)$ and we further assume that the pressure is related to the function $F$ linearly i.e. $P=P_{0}+P_{1} F$ with $P_{0}$ and $P_{1}$ being constants. The constants that appear in the above relations are characteristic plasma parameters and can be expressed in terms of the total current $I_{s}$, the torsion 
$l / T(s)$ and $\beta$. If we take $F=P=0$ which leads to $P_{0}=0$ then this corresponds to the case where the toroidal plasma surface is surrounded by vacuum.

The differential operator that appears in equation (4) can be expressed in terms of the Cartesian coordinates $x(\rho, t)$ and $y(\rho, t)$ as $\mathscr{L}=\nabla^{2}+A_{k_{0}}(\partial / \partial x)$ with $\nabla^{2}=$ $\partial^{2} / \partial x^{2}+\partial^{2} / \partial y^{2}$. From equation (3) we note that the longitudinal component of the current density can be written as

$$
j_{s}=\left(1+\varepsilon_{k} \frac{x}{a}\right)\left[j_{0}-P_{1}\left(-\frac{2 \varepsilon_{k} x}{a}+\varepsilon_{k}^{2} \frac{x^{2}}{a^{2}}+\frac{\varepsilon_{t}^{2}}{a^{2}}\left(x^{2}+y^{2}\right)\right)\right]
$$

where $j_{0}=-\left(P_{1}+\lambda / 2\right)$ is the current density at the central axis $(x=y=0)$ of the toroidal plasma. This central current represents to zero order the average density of the total longitudinal current across the plasma cross section. Similarly from equation (1) we see that the longitudinal component of the magnetic field, $B_{s}$, is given by

$$
B_{s}=\left(1+\varepsilon_{k} \frac{x}{a}\right) B_{0}-\varepsilon_{i} \frac{\rho}{a} \frac{\partial F}{\partial \rho}
$$

where $\rho=\sqrt{x^{2}+y^{2}} ;$ and that $B_{0}=\sqrt{\left\langle B_{s}{ }^{2}\right\rangle}=f_{0}(1+O(\varepsilon)$.

With the effects of toroidicity incorporated the solution of equation (4) can be put in the form

$$
F=F_{s} F_{c}
$$

where

$$
\begin{aligned}
& F_{s}=F_{0}\left[1-\frac{x^{2}}{a^{2}}-\frac{y^{2}}{b^{2}}-\varepsilon_{s}\left(\frac{x}{a}\right)\left(\frac{x^{2}}{a^{2}}-3 \frac{y^{2}}{b^{2}}\right)\right] \\
& F_{c}=1-\nu \frac{x}{a}+\gamma_{1} \varepsilon_{k} \nu \frac{x^{2}}{a^{2}}+\gamma_{2} \varepsilon_{k} \nu \frac{y^{2}}{b^{2}}+\cdots
\end{aligned}
$$

and $F_{0}, \nu, \gamma_{1}$ and $\gamma_{2}$ are constants. The equation for triangular deformation is simply $F_{s}=0$ or

$$
1=\frac{x^{2}}{a^{2}}+\frac{y^{2}}{b^{2}}+\varepsilon_{s}\left(\frac{x}{a}\right)\left(\frac{x^{2}}{a^{2}}-3 \frac{y^{2}}{b^{2}}\right)
$$

where $\varepsilon_{s}$ is the appropriate distortion factor. Figure 2 shows the possible shapes of this cross section. We have used $\varepsilon_{s}=\gamma A_{k_{0}}$ where $\gamma$ is an arbitrary constant, and have assumed that $\varepsilon_{s}$ is of the order of $\varepsilon$ (corresponding to weak deformation) so that terms containing $\varepsilon_{s}$ in the zero order approximation can be ignored relative to unity. When $\varepsilon_{s}=0$ the plasma cross section reduces to that of an ellipse whose major and minor axis are $2 \mathrm{~b}$ and $2 \mathrm{a}$ respectively. The 'singular' points can be readily seen to be: (i) $x=y=0$ (elliptic point) for which $F_{s} / F_{0}=1$. (ii) $x / a=-\left(2 / 3 \varepsilon_{s}\right)$, $y / b=0$ (hyperbolic point) for which $F_{s} / F_{0}=\left(1-4 / 27 \varepsilon_{s}{ }^{2}\right)$. (iii) $x / a=1 / 3 \varepsilon_{s}$; $y / b= \pm\left(1 / \sqrt{3} \varepsilon_{s}\right.$ (two hyperbolic points) for which $F_{s} / F_{0}=\left(1-4 / 27 \varepsilon_{s}^{2}\right)$. The constants $F_{0}, v, \gamma_{1}$ and $\gamma_{2}$ can be determined by substituting equations (19-21) into 


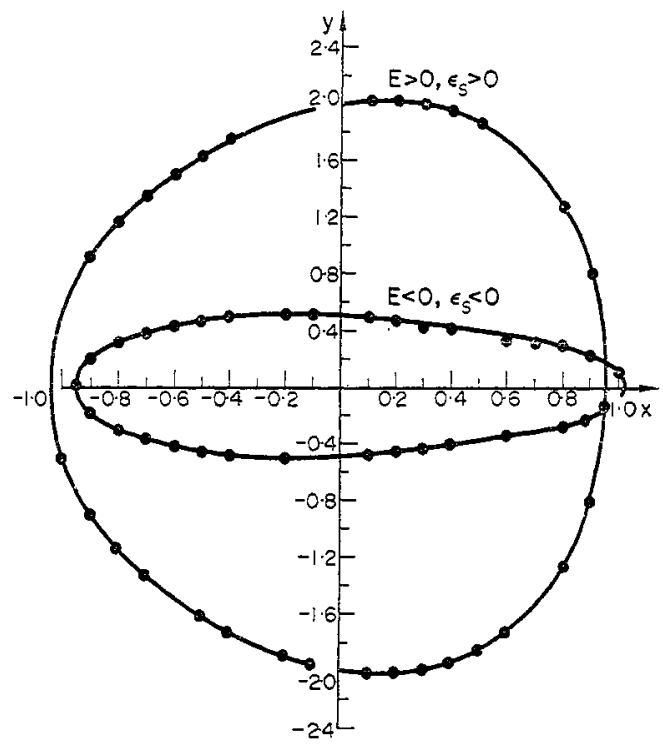

Fig. 2.-Possible shapes of the triangular deformation plasma cross section.

(4); the results are

$$
\begin{aligned}
& F_{0}=\frac{a^{2}\left(\frac{2}{a} B_{0} \varepsilon_{t}-j_{0}\right)}{2\left(1+\frac{a^{2}}{b^{2}}\right)}\left\{1+\frac{\varepsilon_{k}^{2}\left(2 \gamma_{1}+2 \gamma_{2} \frac{a^{2}}{b^{2}}-1\right) \sigma}{\left(3+\frac{a^{2}}{b^{2}}\right)}\right\} \\
& \frac{v}{\varepsilon_{k}}=\frac{\left[\frac{3 \gamma}{a}\left(1-\frac{a^{2}}{b^{2}}\right)+1\right]+2\left(1+\frac{a^{2}}{b^{2}}\right) \sigma}{\left(3+\frac{a^{2}}{b^{2}}\right)+\varepsilon_{k}^{2}\left(2 \gamma_{1}+2 \gamma_{2} \frac{a^{2}}{b^{2}}-1\right) \sigma} \\
& \gamma_{1}=\frac{1}{2}+\frac{2 \varepsilon_{k}^{2}\left(1+6 \frac{a^{2}}{b^{2}}-3 \frac{a^{4}}{b^{4}}\right)\left(\frac{\gamma}{a}\right)+\varepsilon_{t}{ }^{2} \frac{a^{2}}{b^{2}}\left(3+\frac{a^{2}}{b^{2}}\right)}{3 \varepsilon_{k}^{2}\left(1+6 \frac{a^{2}}{b^{2}}+\frac{a^{4}}{b^{4}}\right)} \\
& \gamma_{2}=\frac{-8\left(\frac{\gamma}{a}\right) \varepsilon_{k}^{2}+\frac{b^{2}}{a^{2}} \varepsilon_{t}^{2}\left(3+\frac{a^{2}}{b^{2}}\right)}{2 \varepsilon_{k}^{2}\left(1+6 \frac{a^{2}}{b^{2}}+\frac{a^{4}}{b^{4}}\right)}
\end{aligned}
$$

with

$$
\sigma=\frac{\left(P_{1}-\frac{2 \varepsilon_{t}}{a} B_{0}\right)}{\left(\frac{2 \varepsilon_{t} B_{0}}{a}-j_{0}\right)}
$$


If we define the mean pressure as that averaged over the plasma volume then it can be written as

$$
\langle P\rangle=\frac{1}{V} \int P \mathrm{~d} v \simeq \frac{1}{2} P_{1} F_{0}
$$

similarly we define $\beta=2\langle P\rangle / B_{0}{ }^{2} \simeq P_{1} F_{0} / B_{0}{ }^{2}$. In addition we introduce the nondimensional quantity $G=-\left(k_{t}+1 / q_{(a)}\right)$ with $1 / q=I_{s} / 2 \pi b \varepsilon_{0} B_{0}$ and $I_{s} \simeq \pi a b j_{0}$ and let the quantity $\beta^{*}$ be given by

$$
\beta^{*}=\frac{P_{1}}{\left(\frac{2 \varepsilon_{t} B_{0}}{a}-j_{0}\right)} \simeq \frac{1}{2}\left(1+\frac{a^{2}}{b^{2}}\right) \frac{\beta}{\varepsilon_{0}{ }^{2} G^{2}}[1+O(\varepsilon)] .
$$

In terms of these physical parameters and the ellipticity parameter $E=\left(b^{2}-a^{2}\right)$ / $\left(b^{2}+a^{2}\right)$ of the plasma boundary the constants given by equations $(23-26)$ can now be written as

$$
\begin{aligned}
& F_{0}=\frac{\varepsilon_{0} a B_{0}}{2}(1+E) G\left(1+\frac{\varepsilon_{k}{ }^{2} \Lambda_{2} \sigma}{\left(1+\frac{E}{2}\right)}\right) \\
& \frac{\nu}{\varepsilon_{k}}=\frac{\Lambda_{1}+\sigma}{\left(1+\frac{E}{2}\right)+\varepsilon_{k}{ }^{2} \Lambda \sigma} \\
& \gamma_{1}=\frac{1}{2}+\frac{4\left(\frac{\varepsilon_{s}}{\varepsilon_{k}}\right)\left(1+2 E-2 E^{2}\right)+\frac{\varepsilon_{t}{ }^{2}}{\varepsilon_{k}{ }^{2}}(1-E)(2+E)}{6\left(2-E^{2}\right)} \\
& \gamma_{2}=\frac{(1+E)^{2}\left[-4 \frac{\varepsilon_{s}}{\varepsilon_{k}}(1-E)+\frac{\varepsilon_{t}{ }^{2}}{\varepsilon_{k}{ }^{2}}(2+E)\right]}{4\left(2-E^{2}\right)(1-E)}
\end{aligned}
$$

where

$$
\begin{aligned}
& \Lambda_{1}=\frac{1}{4}\left[1+\left(6 \frac{\varepsilon_{s}}{\varepsilon_{k}}+1\right) E\right] \\
& \Lambda_{2}=\frac{1}{4}\left[\left(2 \gamma_{1}+2 \gamma_{2}-1\right)+\left(2 \gamma_{1}-2 \gamma_{2}-1\right) E\right]
\end{aligned}
$$

and

$$
\sigma=\frac{k_{t}}{G}+\beta^{*}
$$

with

$$
\beta^{*}=\frac{\beta}{(1+E) \varepsilon_{0}^{2} G^{2}}
$$

Figures 3 and 4 show a typical plot of these magnetic surfaces. The solution for the plasma with purely elliptic cross section can be obtained from the above equations by letting $\varepsilon_{s}=0$. For the case of a circular cross section with radius $a\left(\varepsilon_{s}=E=0\right)$ equation (24) yields

$$
\frac{\nu}{\varepsilon_{k}}=\left(\frac{1}{4}+\frac{k_{t}}{G}+\beta^{*}\right)[1+0(\varepsilon)]
$$

which for $I_{s}=0$ further becomes

$$
\frac{\nu}{\varepsilon_{k}}=\left(-\frac{3}{4}+\frac{\beta}{\varepsilon_{0}^{2} k_{t}^{2}}\right)[1+0(\varepsilon)] .
$$




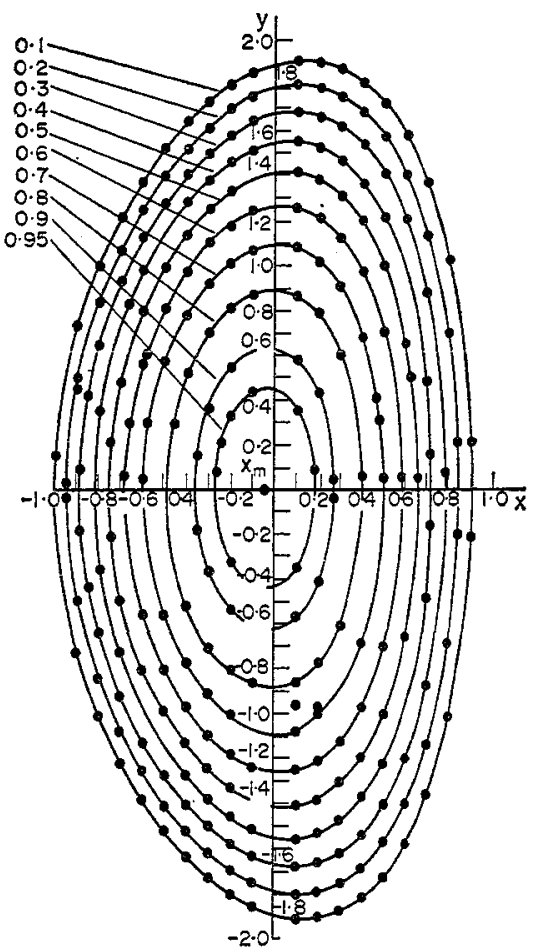

FIG. 3.-Magnetic surfaces in the plasma for $1 / q=0, k_{0}=3, L / 2 \pi T=2.8455, \beta=0.0045$, $\varepsilon_{0}=0.3494 ; \varepsilon_{k}=0.36413, \varepsilon_{s}=0.1, E=0.6$ and different values of $F / F_{0}$. The magnetic axis is displaced at distance $x_{m}=-0.05$ from the central axis of the plasma

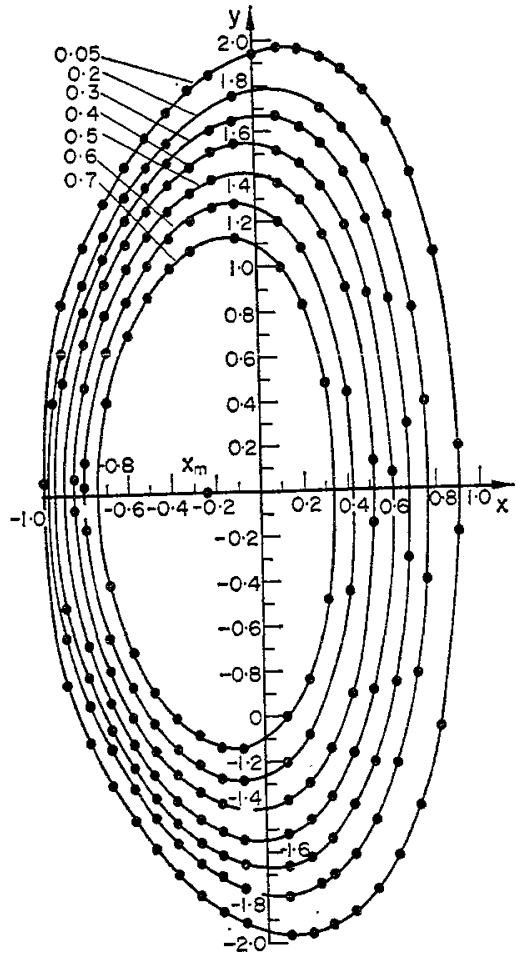

FIG. 4.-Magnetic surfaces in the plasma for $1 / q=0.3, L / 2 \pi T=2.8455, k_{0}=3, \beta=0.084$, $\varepsilon_{0}=0.3494, \varepsilon_{k}=0.36413, \varepsilon_{\mathrm{s}}=0.1, E=0.6$ and different values of $F / F_{0}$. The magnetic axis is displaced at distance $x_{m}=-0.246$ from the central axis of the plasma.

These results agree with those obtained by other authors; for example equation (33) in the limit of zero torsion $\left(1 / T_{s}=0\right)$ reduces to that given in ADAM and MERCIER (1969). Moreover, the MHD equilibria of the French Harmonica $\left(1 / T_{(s)}=0\right.$, $R^{-1}(s)=$ variable, $k_{0} \neq 0$ ) and Tokamak (LAVAL et al., 1971)- $\left(1 / T_{(s)}, R^{-1}(s)=\right.$ constant, $k_{0}=0$ ) with triangular or elliptic cross sections are just special cases of the solutions given by equations (29-32).

Before proceeding to the investigation of the limiting values of $\beta$ associated with these equilibria it would be desirable to briefly examine the geometric implications of the distortion of the plasma cross section. For the non-circular (elliptically or triangularly deformed) plasma cross section with the principal normal to the non-planar magnetic axis of variable torsion and curvature (as described in the image system characterized by two variables $\rho$ and $t=\theta+\delta(s) / 2)$ rotates $\left(-k_{0}\right)$ times along the plasma ring-(in the real system given by $\left(\rho, \theta_{0}, s\right)$ ). For circular cross section plasma with low $\beta$ (the magnetic surfaces are concentric circles)-the effect of the phase angle will not appear in the real system since it remains in the same position as it rotates. Moreover the position of the magnetic axis relative to the central axis will not only change as we go from the image to the real systems but it will also rotate around the central axis. The physical picture can be made clearer when applied to the case of a 
toroidal plasma with non-planar magnetic axis of variable curvature and constant torsion. (See appendix).

ii. Expansion of the solution $\mathrm{F}$ near the magnetic axis

The function $F$ expressed in terms of a system $\bar{\rho}, u$ centered around the magnetic axis can be written as

$$
\frac{F}{F_{0}}=Q+M_{1}\left(\frac{x-x_{m}}{a}\right)^{2}+M_{2}\left(\frac{y}{b}\right)^{2}+N_{1}\left(\frac{x-x_{m}}{a}\right)\left(\frac{y}{b}\right)^{2}+N_{2}\left(\frac{x-x_{m}}{a}\right)^{3}
$$

or

$$
F=F_{m}+(A+B \cos 2 u) \bar{\rho}^{2}+(C \cos u+D \cos 3 u) \bar{\rho}^{3} \text {. }
$$

The point $\left(x_{m}, 0\right)$ is assumed to be the position of the magnetic axis and the remaining quantities in the above equations are defined as follows:

$$
\begin{gathered}
x_{m}-x=\bar{\rho} \cos u ; \quad y=\bar{\rho} \sin u \\
Q_{m}=\frac{F_{m}}{F_{0}}=\left(1-\frac{x_{m}{ }^{2}}{a^{2}}-\varepsilon_{s} \frac{x_{m}{ }^{3}}{a^{3}}\right)\left(1-\nu \frac{x_{m}}{a}+\gamma_{1} \varepsilon_{k} \nu \frac{x_{m}{ }^{2}}{a^{2}}\right) \\
A=\frac{\varepsilon_{0} B_{0} G}{4 a} C_{1} \quad B=\frac{\varepsilon_{0} B_{0} G}{4 a} C_{2} \\
C=\frac{\varepsilon_{0} B_{0} G}{4 a^{2}} C_{3} \quad D=\frac{\varepsilon_{0} B_{0} G}{8 a^{2}} C_{4} \\
C_{1}=M_{1}(1+E)+M_{2}(1-E) \quad C_{2}=M_{1}(1+E)-M_{2}(1-E) \\
C_{3}=3 N_{2}(1+E)+N_{1}(1-E) \quad C_{4}=N_{2}(1+E)-N_{1}(1-E) \\
M_{1}=\left(\gamma_{1} \varepsilon_{k} v-1\right)+3\left(v-\varepsilon_{s}\right)\left(\frac{x_{m}}{a}\right)+6 v\left(\varepsilon_{s}-\gamma_{1} \varepsilon_{k}\right)\left(\frac{x_{m}}{a}\right)^{2} \\
M_{2}=\left(\gamma_{2} \varepsilon_{k} \nu-1\right)+\left(\nu+3 \varepsilon_{s}\right)\left(\frac{x_{m}}{a}\right)-v\left[3 \varepsilon_{s}+\varepsilon_{k}\left(\gamma_{1}+\gamma_{2}\right)\right]\left(\frac{x_{m}}{a}\right)^{2} \\
N_{1}=\left(\nu+3 \varepsilon_{s}\right)-2 v\left[3 \varepsilon_{s}+\varepsilon_{k}\left(\gamma_{1}+\gamma_{2}\right)\right]\left(\frac{x_{m}}{a}\right) \\
N_{2}=\left(\nu-\varepsilon_{s}\right)+4 v\left(\varepsilon_{s}-\gamma_{1} \varepsilon_{k}\right)\left(\frac{x_{m}}{a}\right)
\end{gathered}
$$

We can now return to equations (6a) and (36) to obtain the expression for the transverse flux $\Psi^{\circ}$. It is

$$
\Psi_{0}=(A+B \cos 2 u) \bar{\rho}^{2}+\left(\frac{1}{2 a}\left(\frac{M_{2} N_{2}-M_{1} N_{1}}{M_{1} M_{2}}\right) \frac{\sqrt{1-E_{m}^{2}}}{\left(2+E_{m}\right)} \cos 3 u\right) \bar{\rho}^{3}
$$

and where we readily note that the magnetic surface near the magnetic axis to order $\bar{\rho}^{2}$ corresponds to a small ellipse with an ellipticity given by

$$
E_{m}=t h \eta=\frac{M_{1}(1+E)-M_{2}(1-E)}{M_{1}(1+E)+M_{2}(1-E)} .
$$


By comparing equation (37) with the Mercier's standard form (10) we find that

$$
\begin{aligned}
& \tilde{s}=0 \\
& \tilde{r}=\frac{\left(M_{2} N_{2}-M_{1} N_{1}\right) \sqrt{1-E_{m}^{2}}}{2 a \sqrt{B_{0}} M_{1} M_{2}\left(1+\frac{\varepsilon_{k} x_{m}}{2 a}\right)\left(2+E_{m}\right)} .
\end{aligned}
$$

These two terms along with $\eta, d(s) / 2$ characterize the magnetic surface near the magnetic axis. Also, we can write from equations (6a) and (36) the expression for the flux $\Psi$ as

$$
\begin{array}{r}
\Psi=\frac{\pi B_{s 0}\left|\frac{i_{c 0}}{2 \pi}\right|}{\sqrt{1-E_{m}^{2}}}\left[\left(1+E_{m} \cos 2 u\right) \bar{\rho}^{2}+\left\{\frac{1}{2 a}\left(\frac{3 N_{2}(1+E)+N_{1}(1-E)}{M_{1}(1+E)+M_{2}(1-E)}\right) \cos u\right.\right. \\
\left.\left.+\frac{1}{2 a}\left(\frac{N_{2}(1+E)-N_{1}(1-E)}{M_{1}(1+E)+M_{2}(1-E)}\right) \cos 3 u\right\} \bar{\rho}^{3}\right] .
\end{array}
$$

If we now compare this with Solevev'ev and Shafranov's normal form (11) we find that

$$
\begin{aligned}
& \alpha_{1}=\frac{1}{2 a}\left(\frac{3 N_{2}(1+E)+N_{1}(1-E)}{M_{1}(1+E)+M_{2}(1-E)}\right) \\
& \alpha_{2}=0 \\
& \ddot{\alpha}_{3}=\frac{1}{2 a}\left(\frac{N_{2}(1+E)-N_{1}(1-E)}{M_{1}(1+E)+M_{2}(1-E)}\right) \\
& \alpha_{4}=0
\end{aligned}
$$

The longitudinal component of the magnetic field $B_{s 0}$ and the current density of $j_{s 0}$ near the magnetic axis can be obtained from equations (17) and (18). The result is

$$
\begin{aligned}
& B_{s 0}=B_{0}\left(1+\varepsilon_{k} \frac{x_{m}}{a}\right) \\
& j_{s 0}=j_{0}\left(1+\varepsilon_{k} \frac{x_{m}}{a}\right)\left(1+q \beta^{*} G W\right)
\end{aligned}
$$

where the quantity $W$ is given by;

$$
W=\frac{2 \varepsilon_{k} x_{m}}{a}-\left(\varepsilon_{k}^{2}+\varepsilon_{t}^{2}\right) \frac{x_{m}^{2}}{a^{2}} .
$$

MERCIER's necessary condition (1964) for stability and SoLVEV'EV's necessary and sufficient conditions (1969) depend on the above quantities as well as on the curvature and torsion of the magnetic axis, on the rate $d^{\prime}(s) / 2$ at which the cross section turns, and on the ratio $j_{s 0} / B_{s 0}$. As mentioned earlier these stability criteria will be examined in a subsequent paper. 
Following the same procedure we find that the rotational transform near the magnetic axis, $i_{c 0} / 2 \pi$, can be put in the form

$$
\frac{i_{e 0}}{2 \pi}=-k_{0}+G \sqrt{1-E_{m}^{2}}\left(\beta^{*} W-1\right)
$$

and on the boundary of the plasma it is given by

$$
\frac{i_{c b}}{2 \pi}=-k_{0}+G \sqrt{\left(1-v^{2}\right)\left(1-E^{2}\right)}(1+O(\varepsilon)) .
$$

These expressions agree with those given in ADAM and MERCIER (1969) for circular cross section with planar magnetic axis. For $v=1$ equations (45) and (46) reduce to $i_{c o} / 2 \pi=-k_{0}+G \sqrt{\frac{8}{3}\left(1-E^{2}\right)}$ and $i_{c b} / 2 \pi=-k_{0}$ respectively while for $\nu \ll 1$ the two transforms become equal and have the value $i_{c 0} / 2 \pi=i_{c o} / 2 \pi=-k_{0}+G \sqrt{1-E^{2}}$.

The expression for $V_{0}^{\prime \prime}$ for the present case can now be calculated from formula (16), (38) and (42); the result is

$$
\begin{array}{r}
\left.V_{0}^{\prime \prime}=\frac{-L}{\pi B_{s 0}^{2} \sqrt{1-\frac{C_{2}^{2}}{C_{1}^{2}}}\left(\frac { 1 } { 2 } \left(\sum_{k}\left|A_{k_{0}}\right|^{2}\right.\right.}-\frac{C_{2}}{C_{1}} \sum_{k} A_{k} A_{2 k_{0}-k} \cos \left(\phi_{k}-\phi_{2 k_{0}-k}-2 \phi_{k_{0}}\right)\right) \\
\left.-\left(\frac{\varepsilon_{t} C_{2}}{a C_{1}}\right)^{2}-\frac{A_{k_{0}}}{2 a}\left(\frac{C_{3}\left(2 C_{1}-C_{2}\right)-3 C_{2} C_{4}}{C_{1}\left(C_{1}+C_{2}\right)}\right)\right\}
\end{array}
$$

In the special case when the displacement of the magnetic axis is weak, say $\left(x_{m} \mid a\right) \sim \varepsilon_{k}$ which corresponds to $\beta^{*} \leqslant 1$ and $\nu \simeq \varepsilon_{k} \beta^{*} /(1+E / 2) \sim \varepsilon$, we find that

$$
\begin{gathered}
M_{1}=M_{2} \simeq-1, \quad N_{1} \simeq\left(\nu+3 \varepsilon_{s}\right), \quad \bar{N}_{2} \simeq\left(\nu-\varepsilon_{s}\right), \quad E_{m} \sim E, \quad W \simeq 0, \\
j_{s 0} \sim j_{0}, \quad B_{s 0} \sim B_{0}, \quad \frac{i_{c 0}}{2 \pi}=-k_{0}-G \sqrt{1-E^{2}}, \quad \sqrt{B_{s 0}} \tilde{r}=\frac{2 \varepsilon_{s}}{a} \frac{\sqrt{1-E^{2}}}{(2+E)}, \\
\alpha_{1}=-\frac{1}{2 a}\left(\nu(E+2)-3 \varepsilon_{s} E\right), \quad \alpha_{3}=-\frac{1}{2 a}\left(\nu E+\varepsilon_{s}(E-2)\right) .
\end{gathered}
$$

The singular points (the positions of the separatrix) of the curve of the cross section with $\mathrm{d}(s) / 2=0$ are given by the equations $\partial F / \partial x=0$ and $\partial F / \partial y=0$ as (i) $\left(x_{s 1} / a\right)=$ $2 / 3\left(v-\varepsilon_{s}\right)$ and $\left(y_{s 1} / b\right)=0$ (hyperbolic point), (ii) $\left(x_{s 2} / a\right)=1 /\left(v+3 \varepsilon_{s}\right)$ and $\left(y_{s 2, s} \mid b\right)^{2}=\left(9 \varepsilon_{s}-v\right) /\left(\nu+3 \varepsilon_{s}\right)^{2}$ (two hyperbolic points), at which $\left(F / F_{0}-Q_{m}\right)=$ $-4 / 27\left(v-\varepsilon_{s}\right)^{2}$ and

$$
\Psi=\frac{4 \pi B_{0} a^{2}\left(\frac{i_{c 0}}{2 \pi}\right)}{27\left(\varepsilon_{s}-\nu\right)^{2}} \sqrt{\frac{1+E}{1-E}} .
$$

In this case expression (47) takes the form

$$
\begin{array}{r}
V_{0}^{\prime \prime}=\frac{-L}{\pi B_{s 0}^{2} \sqrt{1-E^{2}}}\left\{\frac{1}{2}\left(\sum_{k}\left|A_{k_{0}}\right|^{2}-E \sum_{k} A_{k_{0}} A_{2 k_{0}-k} \cos \left(\phi_{k}-\phi_{2 k_{0}-k}-2 \phi_{k_{0}}\right)\right)\right. \\
\left.-\frac{E^{2} \varepsilon_{t}^{2}}{a^{2}}+\left(\frac{2 v A_{k_{0}}}{a}\right)(1-E)\right\} .
\end{array}
$$


In order to obtain the condition for existence of the magnetic well (i.e. $V_{0}^{\prime \prime}<0$ ), we need to calculate numerically the Fourier components of the relative curvature appearing in expression (48) for the class of equilibria that belongs to the non-planar magnetic axis. As an example, these components are calculated in the appendix for a particular class of equilibria of non-planar magnetic axis closed curve of constant torsion turned three times around the magnetic axis characterized by $L / 2 \pi T=$ 2.84549 and $k_{0}=3$. In this case, the expression for $V_{0}^{\prime \prime}$ given by (48) takes the form for low values of $\beta$

$$
V_{0}^{\prime \prime}=\frac{-L}{\pi B_{s 0}^{2} \sqrt{1-E^{2}}}\left[\frac{1}{2}(0.30885-0.1526 E)-\frac{E^{2} \varepsilon_{t}^{2}}{a^{2}}+(0.36413)\left(\frac{2 v}{a}\right)(1-E)\right] \text {. }
$$

We note that expression (49) does not contain any $\varepsilon_{s}$ terms thereby indicating that the triangular deformation has no effect on $V_{0}^{\prime \prime}$. The condition for existance of a magnetic well $\left(V_{0}^{\prime \prime}<0\right)$ is that the terms in the square bracket be positive. We assume first that the configuration of magnetic surface contains only a single magnetic axis (i.e. $\nu<1$ and positive). For horizontally elliptic cross section $(E<0)$ the square bracket is positive $\left(V_{0}^{\prime \prime}<0\right)$ and the result is a magnetic well. For vertically elliptic cross section $(E>0)$, the square bracket may take on a negative value $\left(V_{0}^{\prime \prime}>0\right)$ leading to a magnetic hill. For circular cross section $(E=0)$ the square bracket has an absolutely positive value, thus $V_{0}^{\prime \prime}<0$ and the result is a magnetic well. These results are valid in the presence or absence of longitudinal current.

It might be noted that the equilibrium examined here belongs to a particular type of stellarator with or without Ohmic heating $\left(1 / T(s) \neq 0 ; j_{s 0} \neq 0\right.$ or $\left.j_{s 0}=0\right)$, and the equilibrium solution for Tokamak $\left(1 / T(s)=0 ; j_{s 0} \neq 0 ; 1 / R(s)=\right.$ constant $)$ and the French Harmonica $\left(1 / T(s)=0 ; j_{s 0} \neq 0 ; 1 / R(s)=\right.$ variable $)$ are just special cases of the above solution.

\section{iii. Limiting values of $\beta$ for equilibrium}

The limiting value of $\beta$ can be estimated from the condition which corresponds to the appearance of a new magnetic axis on the boundary of the toroidal plasma (LAval et al., 1971). A single inward magnetic axis exists inside this boundary when $v<1$ and that corresponds to a maximum pressure profile. A second new outward magnetic axis appears when $\nu \geqslant 1$ and this corresponds to a minimum pressure profile in the configuration. One is usually interested in a plasma configuration in which the magnetic surface encircles a single magnetic axis. If we let $x=x_{m}, y=0$ be the coordinates of the magnetic axis relative to the central axis which is determined from the condition $(\nabla F)_{F=0}=0$, and assume that the displacement of the magnetic axis is toward the center of the torus i.e. $\left(x_{m} / a\right)<0$ then to zero order approximation we get $\left(x_{m} / a\right) \approx 1-\sqrt{1+3 v^{2}} / 3 v$. If we further define the limiting $\beta$ for $\nu \sim 1$ as

with

$$
\beta_{P \max } \approx(1+E) \varepsilon_{0}{ }^{2} G^{2} \beta_{P \max }^{*}
$$

$$
\beta_{P \max }^{*}=\frac{1}{\varepsilon_{k}}\left(1+\frac{E}{2}\right)-\frac{1}{4}\left[1+\left(\frac{6 \varepsilon_{s}}{\varepsilon_{k}}+1\right) E\right]-\frac{k_{t}}{G}
$$

then it is clear that the case $1 / q=-k_{t}$ corresponds to the resonance case at which 
$G=0$ and $\beta=0$. To the zero order approximation these results reduce to

$$
\begin{aligned}
& \beta_{P_{\max }}^{*} \approx \frac{\left(1+\frac{E}{2}\right)}{\varepsilon_{k}}(1+0(\varepsilon)) \sim \frac{1}{\varepsilon} \\
& \beta_{P_{\max }} \approx \frac{\varepsilon_{0}^{2} G^{2}}{\varepsilon_{k}}(1+E)\left(1+\frac{E}{2}\right) \sim \varepsilon
\end{aligned}
$$

We note that equations (52) and (53) do not contain any terms related to the triangular deformation of the plasma cross section. This implies that the influence of this kind of distortion is weak and can be neglected to lowest order. On the other hand elliptic deformations have a significant effect on the limiting $\beta$, vertically elongated $(E>0)$ elliptic cross sections are preferable for equilibria at high $\beta$ values. This conclusion agrees with that of LAVAL et al. (1971) for the case of a Tokamak with triangularly deformed plasma cross section. For the low $\beta\left(\beta^{*} \leqslant 1\right)$ case we find that $\beta_{\text {max }} \approx$ $\varepsilon_{0}{ }^{2} G^{2}(1-E) \sim \varepsilon^{2}$. If we substitute $1 / T=k_{0}=0, L=2 \pi R$ and $A_{0}=1 / R$ in equations (52) and (53) we obtain the limiting values of $\beta$ for Tokamak plasmas i.e.

$$
\begin{aligned}
& \beta_{P_{\max }}^{*} \simeq \frac{R}{a} 1+\frac{E}{2} \\
& \beta_{P_{\max }} \simeq \frac{a}{R}\left(\frac{R j_{0}}{2 B_{0}}\right)^{2}(1+E)\left(1+\frac{E}{2}\right) .
\end{aligned}
$$

In the interesting case where the longitudinal current is absent (as might exist in a particular type of stellarator without ohmic heating) equation (53) becomes

$$
\beta_{P_{\max }} \approx \frac{\varepsilon_{0}^{2} k_{t}^{2}}{\varepsilon_{k}}(1+E)\left(1+\frac{E}{2}\right)
$$

As an application, we have carried out numerical calculations from equation (53) for a particular type of stellarator configuration namely that of non-planar magnetic axis of variable curvature and constant torsion characterized by $L / 2 \pi T=2.84549$ and $k_{0}=3$ (The properties of this configuration are studied in detail in the appendix.) This geometry is further characterized by an inverse aspect ratio of $\varepsilon_{0}=0.1$ and with a longitudinal current through the non-circular cross section plasma in forward and backward directions. The results are given in Fig. 5 which shows $1 / q$ vs the limiting value of $\beta$ for equilibrium and also defines the domains of equilibrium. We readily see that if all the curves are extended they will intersect at a point which corresponds to the resonance case at a value of $1 / q=-\left(k_{0}-L / 2 \pi T\right)=-0.1545$ for which $G=0$ and $\beta_{P \max }=0$. We also note that for $1 / q \neq 0$ equilibrium is possible without constant torsion. In the absence of longitudinal current equilibrium exists at a low value of $\beta$ (limited between from zero to $1.4 \times 10^{-5}$ for $E=-0.99$ and from zero to $7.05 \times 10^{-3}$ for $E=+0.99$ with $\varepsilon_{0}=0.1$ ). The class of equilibrium considered here has been examined by RIzK and KAMMASH (1973) for a weak flat and non-flat 


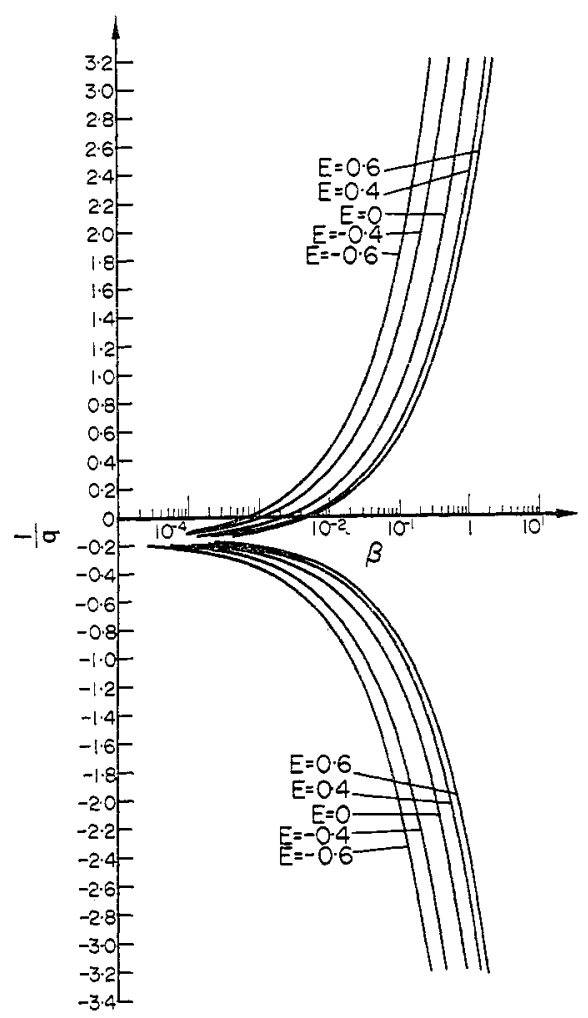

FIG. 5.-Plot of $\beta_{P_{\text {max }}}$ vs $1 / q$ for $L / 2 \pi T=2.8455, k_{0}=3$, and $\varepsilon_{0}=0.1$.

profiles of a current flowing in the forward and backward directions through a circular plasma cross section where it has been shown that the effect of the nonuniformity in current density profile is very weak and can be neglected. Finally it should be pointed out that the class of equilibria considered here belongs to a non-planar closed magnetic axis of constant torsion characterized by $L / 2 \pi T=$ 2.84549 and $k_{0}=3$.

\section{CLASSICAL DIFFUSION}

In this section, we discuss the classical diffusion of non-axisymmetric toroidal plasma in the presence of the non-planar magnetic axis of a variable curvature and torsion and with non-circular (elliptically or triangularly deformed) cross section. The magnetic toroidal configuration considered here represents a particular type of stellarator.

The general expression for the average diffusion velocity across a magnetic surface $\psi=\psi_{0}=$ constant is given by (MASCHKE, 1971)

$$
\frac{\left\langle V_{D}\right\rangle}{V_{s p}}=1+\int_{\psi_{0}} \frac{\mathrm{d} s}{|\nabla \Psi|}\left(\eta_{\|} j_{\|}{ }^{2}-\mathbf{j} \cdot \mathbf{E}_{\mathrm{ext}}\right) / \int_{\psi_{0}} \frac{\mathrm{d} s}{|\nabla \Psi|} \eta_{\perp} \frac{(\nabla P)^{2}}{B^{2}}
$$

where $V_{s p}$ is the resistive diffusion velocity (SPITZER, 1962) in a straight plasma column 
with the same magnetic surfaces cross section as considered here, and $\eta_{\|} ; \eta_{\perp}$ are the resistivities parallel and perpendicular to the magnetic field. The quantity $\mathbf{E}_{\text {ext }}$ is the externally applied toroidal electric field.

Near the magnetic axis of the magnetic surface of the toroidal plasma configuration, expression (47) reduces to

$$
\frac{\left\langle V_{D}\right\rangle}{V_{s p}}=1+\frac{\eta_{1}}{\eta_{\perp}} \frac{\int_{0}^{L} \mathrm{~d} s\left[B_{s 0} Z_{(s)} Z_{(s)}^{*}+j_{s 0} H_{(s)}\right]}{8 P_{0}^{\prime 2} \int_{0}^{L} \mathrm{~d} s \frac{\cosh \eta_{(s)}}{B_{s 0}{ }^{2}}}
$$

with

$$
H_{(s)}=\frac{4 P_{0}^{\prime}}{B_{s 0}^{3}\left|i_{c 0}\right|}\left[\frac{1}{2} j_{s 0}+B_{s 0} K_{(s)}\left(\cosh \eta_{(s)}-1\right)\right] .
$$

The function $Z(s)$ is the solution of equation (43) of MERCIER (1964) which is given (LUC et al., 1974) by

$$
Z(s)=\frac{2 i L P_{0}^{\prime}}{\pi B_{s 0}^{3 / 2}} \sum_{l=-\infty}^{l=+\infty} \frac{\cosh (\eta / 2) A_{k_{0}-l} \mathrm{e}^{i \delta_{l 1}}-\sinh (\eta / 2) A_{\hat{k}_{0}+l} \mathrm{e}^{-i \delta_{l 2}}}{\frac{L K}{2 \pi}-l}
$$

where $\delta_{l_{1}}=\varphi_{k_{0}}-\varphi_{k_{0}-l}+2 \pi l s / L$, and $\delta_{l_{2}}=\varphi_{k_{0}}-\varphi_{k_{0}+l}-2 \pi l s / L$. As may be seen from equation (59) there are singularities in the function $Z(s)$ when the rotational transform near the magnetic axis given by equation (7a) has an interger value i.e. $L K / 2 \pi=l_{\text {res }}+\varepsilon$ with $|\varepsilon| \ll 1$. The interesting case is that of the resonance $L K / 2 \pi=$ $l=l_{\text {res }}=0$ at which the coefficient $A_{l_{0}}$ appears to dominate.

For the resonant case, we can neglect all terms $l \neq 0$ and substituting from equation (59) into equation (58) for the case $L K_{(s)} / 2 \pi \ll 1$ with the class of equilibria given by $\eta(s)=$ constant, $K(s)=\sqrt{1-E_{m}^{2}} \Lambda=$ constant with $\Lambda=\left(j_{s 0} / 2 B_{s 0}\right.$ $\left.\varepsilon_{t} / a\right)$; we get

$$
\frac{\left\langle V_{D}\right\rangle}{V_{s p}}=1+\frac{2 \eta_{i 1}}{\eta_{\perp}}\left\{\frac{\varepsilon_{k}^{2}}{a^{2}\left(1+E_{m}\right) \Lambda^{2}}+\frac{j_{s 0}\left[\Lambda\left(1-\frac{1}{2} \sqrt{1-E_{m}^{2}}+\frac{\varepsilon_{t}}{2 a}\right]\right.}{4 \pi P_{0}^{\prime}\left|\frac{-k_{0}}{\sqrt{1-E_{m}^{2}}}+\frac{L \Lambda}{2 \pi}\right|}\right\} .
$$

If the displacement of the magnetic axis is weak (i.e. $x_{m} / a \sim \varepsilon_{k} ; \beta^{*} \leqslant 1$; $\nu \sim \varepsilon_{k} ; M_{1}=M_{2} \simeq-1 ; W=0 ; j_{s 0} \sim j_{0} ; B_{s 0} \sim B_{0} ; E_{m} \sim E$ and $\left.\Lambda \simeq \varepsilon_{0}|a| G \mid\right)$ equation (60) takes the form

$$
\frac{\left\langle V_{D}\right\rangle}{V_{s p}}=1+\frac{2 \eta_{1}}{\eta_{\perp}}\left[\frac{\varepsilon_{k}^{2}}{\varepsilon_{0}^{2} G^{2}(1+E)}-\frac{\varepsilon_{0}^{2}(1+E)}{2 \beta_{0}} \frac{1}{q}|G|\left\{\left(1-\frac{1}{2} \sqrt{1-E^{2}}\right)-\frac{k_{t}}{2|G|}\right\}\right]
$$

where $\beta_{0}$ is the value of $\beta$ on the magnetic axis. The above relation gives the expression for $\left\langle V_{D}\right\rangle$ near the non-planar magnetic axis of variable torsion and curvature 
of the non-circular cross section toroidal plasma through which a current with flat density profile flows (the case of a particular type of stellarator with Ohmic heating). It also includes the resonance case at which $1 / q=-k_{t}$ for which $G=0$ and $\beta=0$. In the absence of longitudinal current density, formula (61) takes the form

$$
\frac{\left\langle V_{D}\right\rangle}{V_{s p}}=1+\frac{2 \eta_{1}}{\eta_{\perp}} \frac{\varepsilon_{k}^{2}}{\varepsilon_{0}^{2} k_{t}^{2}(1+E)}
$$

at which resonance exists at $k_{0}=\alpha(L) / 2 \pi$. From equation (61), we can also get the expression for $\left\langle V_{D}\right\rangle$ near the magnetic axis for the French Harmonica $(1 / T=0$, $1 / R(s)=$ variable, $k_{0} \neq 0$ ) as

$$
\begin{aligned}
\frac{\left\langle V_{D}\right\rangle}{V_{s p}}=1+\frac{2 \eta_{\|}}{\eta_{\perp}} & \left\{\frac{\varepsilon_{k}{ }^{2}}{\varepsilon_{0}{ }^{2}\left(k_{0}+\frac{1}{q}\right)^{2}(1+E)}\right. \\
& \left.-\frac{\varepsilon_{0}^{2}(1+E)}{2 \beta_{0}} \frac{1}{q}\left(\frac{1}{q}+k_{0}\right)\left[\left(1-\frac{1}{2} \sqrt{1-E^{2}}\right)-\frac{k_{0}}{2\left(k_{0}+\frac{1}{q}\right)}\right]\right\}
\end{aligned}
$$

and for Tokamak $\left(1 / T=0, k_{0}=0, A_{K_{0}}=1 / R(s)=2 \pi / L\right)$ as

$$
\frac{\left\langle V_{D}\right\rangle}{V_{s p}}=1+\frac{2 \eta_{1}}{\eta_{\perp}}\left[\left(\frac{2 B_{0}}{R j_{0}}\right)^{2} \frac{1}{1+E}-\left(\frac{a}{R}\right)^{2}\left(\frac{R j_{0}}{2 B_{0}}\right)^{2}\left(\frac{1+E}{2 \beta_{0}}\right)\left(1-\frac{1}{2} \sqrt{1-E^{2}}\right)\right]
$$

which are just special cases of expression (61). The first term in the square brackets with $E=0$ corresponds to the PFIRSCH-SCHLÜTER (1962) regime. We note from (62) that the second term decreases by increasing $k_{t}$ and the total diffusion velocity also decreases by increasing $k_{t}$ (for this case the maximum value of $\beta$ increases also). The effect of triangular deformation of the plasma cross section on the diffusion velocity exists only for high values of $\beta$; this may be readily seen from equation (61) which contains no $\varepsilon_{s}$ dependent terms. The effect of ellipticity on the diffusion velocity can be discerned from the various terms inside the square bracket of equation (61). We note that the first term decreases for increasing $E$ while the two terms in the curly brackets are positive for positive values of $1 / q$. Therefore, both the second and third terms in the square bracket increase for increasing $E$, leading to the conclusion that the diffusion velocity $\left\langle V_{D}\right\rangle$ decreases for the vertically elongated $(E>0)$ elliptic cross section; a geometry which is also preferable for equilibrium.

\section{CONCLUSION}

We have examined the MHD equilibrium and classical diffusion of a non-axisymmetric toroidal plasma with non-circular cross section (elliptically or triangularly deformed) characterized by non-planar magnetic axis with variable curvature and torsion, and through which flows a longitudinal current of flat density profile in forward and backward directions. We have found that the effect of triangular deformation on the limiting value of $\beta$ for equilibrium and on the classical diffusion 
(for low $\beta$ ) to be weak and negligible; and have also found that a vertically elongated $(E>0)$ elliptic cross section is preferable for equilibrium and with reduced diffusion velocity $\left\langle V_{D}\right\rangle$. We have also concluded that equilibrium does not exist for high values of $\beta$ (corresponding to $y \geqslant 1$ at which a new magnetic axis is supposed to appear) in the absence of longitudinal current. In addition, we have shown that in the absence or presence of longitudinal current, a magnetic well $\left(V_{0}^{\prime \prime}<0\right)$ obtains for horizontal elliptic $(E<0)$ and circular $(E=0)$ cross sections while a magnetic hill $\left(V_{0}^{\prime \prime}>0\right)$ may obtain for a vertically elliptic $(E>0)$ cross section.

Acknowledgement-This work is supported in part by the U.S. Atomic Energy Commission.

\section{REFERENCES}

Adam J. C. and Mercter C. (1969) Plasma Physics and Controlled Fusion Research, IAEA 1, 199. Glasser A. H., FrIeman E. A., and YoshIKAWA S. (1973) Princeton University Report MATT-985, June.

Laval G., Luc H., Maschke E. K., Mercier C., and Pellat R. (1971) Plasma Physics and Controlled Fusion Research, IAEA, CN-28/F-12.

LuC H., MAschke E. K., Mercier C., Misguich and Ruzk H. M. (1974) Plasma Physics 5, 455.

Maschke E. K. (1971) Pasma Phys. 13, 905.

MERCIER C. (1963) Nucl. Fusion 3, 89.

MERCIER C. (1964) Nucl. Fusion 4, 213.

MERCIER C. (1966) Plasma Physics and Controlled Fusion Research, IAEA 417.

NÜBERNBERG J. (1972) Nucl. Fusion 12, 383.

PhFisch D. and Schluter A. (1962) Max Planck Institute-Report MPI/PA/7.

RIZK H. M. and KAMMASH T. (1973), Not published.

Shafranov V. D. (1964) Nucl. Fusion 4, 232.

Shafranov V. D. (1968) Proceedings of the First All-Union Conference on Plasma Confinement Enclosed Magnet Systems, Moscow, FLAN, 15-19 April 1968.

Solev'ev L. S., Shafranov V. D., and YurChenko E. J. (1969) Nucl. Fusion Suppl. 25.

Solev'ev L. S. and Shafranov V. D. (1970) Reviews of Plasma Physics, (Edited by M. A. Shafranov

V. D. (1970) Reviews of Plasma Physics, (Edited by M. A. Leontovich) Consultants Bureau, New York, Vol. 5.

SoLOV'EV L. S. (1968) Sov. Phys. JETP 26, 400.

Solov'ev L. S. (1968) Sov. Phys. JETP 26, 1167.

Soloviev L. S. (1969) Sov. Phys. Dokl. 13, 1050.

SPITZER Jr. (1962) Physics of Fully Ionized Gases, 2nd Edition, p. 43. Interscience, New York.

\section{APPENDIX}

The parametric equations for a closed non-planar magnetic axis characterized by variable curvature and constant torsion; are

$$
\begin{aligned}
& \frac{x(v)}{T}=\mu_{0} \sin v+\mu_{1} \sin 4 v+\mu_{2} \sin 2 v+\mu_{3} \sin 7 v+\mu_{4} \sin 5 v \\
& \frac{y(v)}{T}=\mu_{0}(1-\cos v)+\mu_{1}(1-\cos 4 v)-\mu_{2}(1-\cos 2 v)-\mu_{4}(1-\cos 5 v) \\
& \frac{z(v)}{T}=\mu_{5}\left(\sin 3 v-\frac{1}{2} \sin 6 v\right)
\end{aligned}
$$

with

$$
\mu_{0}=-2.763, \mu_{1}=0.0301 ; \mu_{2}=0.1939 ; \mu_{3}=-0.0277 ; \mu_{\mathrm{s}}=0.0562, \text { and } \mu_{5}=0.1556 .
$$

From these equations we obtain

$$
\frac{1}{T}\left(\frac{\mathrm{d} s}{\mathrm{~d} v}\right)=[8.1329+0.9989 \cos 3 v-0.4668 \cos 6 v]^{1 / 2} .
$$


The curvature of this curve is given by

$$
\begin{aligned}
\frac{T}{R(s)} & =\frac{0.2415[-13.2039+63.131 \cos 3 v+2.9966 \cos 6 v-0.9335 \cos 9 v]}{[8.1329+0.9989 \cos 3 v-0.4668 \cos 6 v]^{3 / 2}} \\
& =T \sum_{k=-\infty}^{\infty} A_{k} \exp i\left(\frac{2 \pi k s}{L}+\varphi_{k}\right)
\end{aligned}
$$

Figure (A-1) shows the projection in the $(X-Y)$ plane of this closed curve whose local geometry is a torus inside the plasma. Numerical calculations have been carried out for this curve with the characteristic value $L / 2 \pi T=2.8455$ and resonance index $k_{0}=3$ (The integer resonance index $k_{0}$ was chosen as near as possible to $L / 2 \pi T$ ), and it was found that the non-circular cross section with the principal normal to the magnetic axis as characterized by the unit vector $\mathbf{n}=\left(n_{x}, n_{z}, n_{z}\right)$ in the image system $\left(\rho, t=\varphi_{k_{0}}+\theta+2 \pi k_{0} s / L\right)$ rotates $-k_{0}(=-3)$ times in the real system $\left(\rho, \theta_{0}=\theta+\right.$ $s / T, s)$ along the plasma ring.

Figure (A-2) shows the projection of both the non-planar closed curve of $L / 2 \pi T=2.84549$ with $1 / T=1$, and its principle normal (represented by the broken curve) in the $(X-Y)$ plane.

Figure (A-3) gives a plot of $\alpha\left(=\tan ^{-1} n_{z} / \sqrt{\left.n_{x}^{2}+n_{y}^{2}\right)}\right.$ the angle between the principle normal $n$ and its projection in the $(X-Y)$ plane, vs the curvilinear distance ' $s$ ' and shows that the principle normal rotates three times around both the central and magnetic axes. For this particular non-planar closed curve the following integrals which enter in the calculations of both the magnetic well function $V_{0}^{\prime \prime}$ and SOLOV'EY's necessary and sufficient criteria of stability 1968 and 1969 near the magnetic axis have been numerically obtained:

$$
\begin{aligned}
\left\langle\frac{1}{R^{2}(s)}\right\rangle & =\frac{1}{L} \int_{0}^{L} \mathrm{~d} s \frac{1}{R^{2}(s)}=\sum_{k=-\infty}^{+\infty}\left|A_{k}\right|^{2}=0.30885 \\
\left\langle\frac{\cos \mathrm{d}(s)}{R^{2}(s)}\right\rangle & =\frac{1}{L} \int_{0}^{L} \mathrm{~d} s \frac{\cos \mathrm{d}(s)}{R^{2}(s)}=\sum_{k=-\infty}^{+\infty} A_{k} A_{2 k_{0}-k} \cos \left(\phi_{k}-\phi_{2 k_{0}-k}-2 \phi_{k}\right)=0.1526 \\
\left\langle\frac{\cos \frac{\mathrm{d}(s)}{2}}{R(s)}\right\rangle & =\frac{1}{L} \int_{0}^{L} \mathrm{~d} s \frac{\cos \frac{\mathrm{d}(s)}{2}}{R(s)}=A_{k}=0.36413 \\
\left\langle\frac{\sin \frac{d(s)}{2}}{R(s)}\right\rangle & =\frac{1}{L} \int_{0}^{L} \mathrm{~d} s \frac{\sin \frac{\mathrm{d}(s)}{2}}{R(s)}=0
\end{aligned}
$$

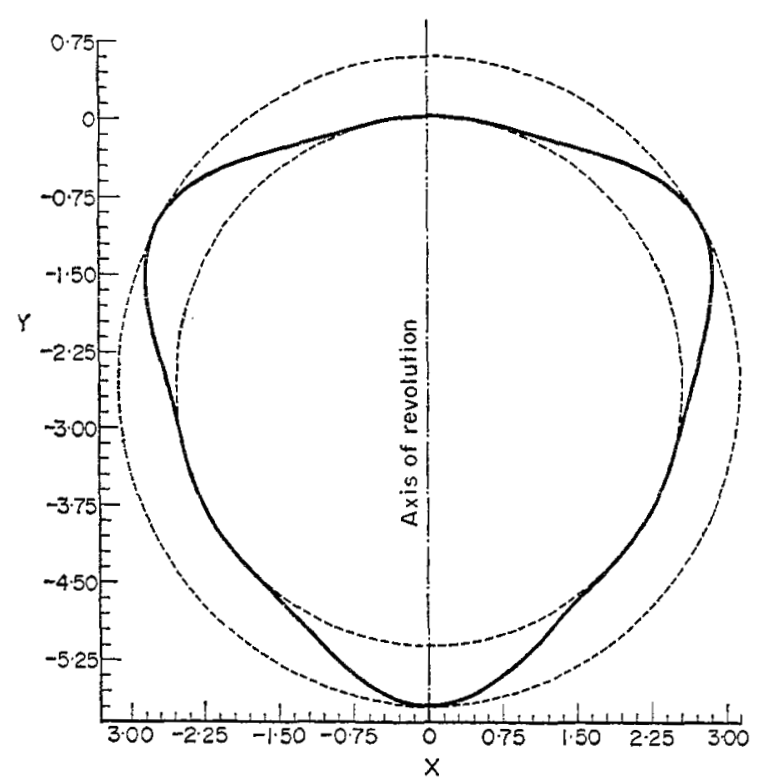

FIG. A-1.-Local geometry of the non-planar closed curve of characteristic $L / 2 \pi T=$ $2 \cdot 84549$ with $1 / T=1$. 


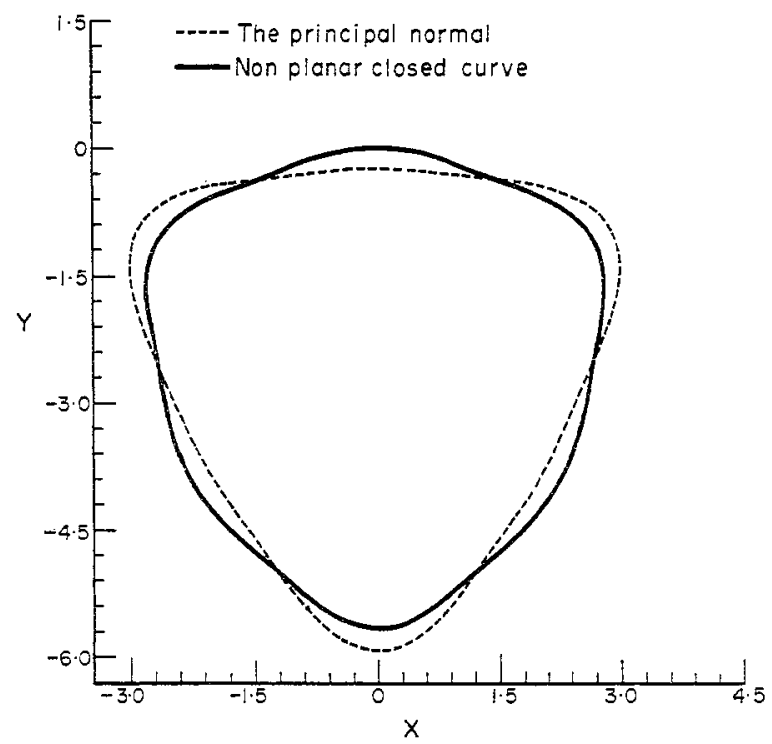

FIG. A-2.-Projection of both the closed non-planar curve of characteristic $L / 2 \pi T=$ 2.8455 with $1 / T=1$ and its principal normal in the $(X-Y)$ plane.

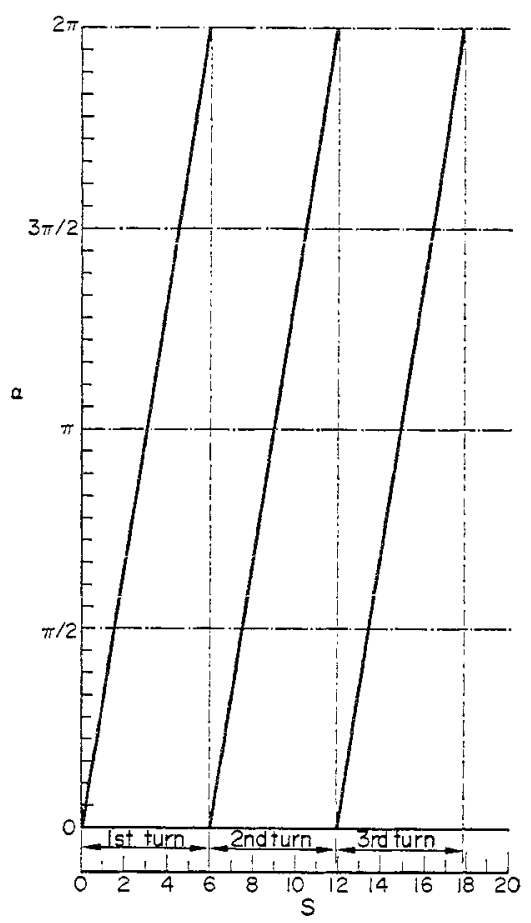

FIG. A-3.-Plot of the angle $\alpha\left(=\tan ^{-1} n_{z} / \sqrt{n_{x}^{2}+n_{y}{ }^{2}}\right)$ between the principal normal $\mathrm{n}$ and its projection in the $(\mathrm{X}-\mathrm{Y})$ plane versus the curvilinear coordinate $s$. 